\title{
Clinico-radio-pathological Features and Biological Behavior of Breast Cancer in Young Indian Women: A Prospective Study
}

\author{
Rashmi Sudhir $^{1} \quad$ Kamala Sannapareddy ${ }^{1} \quad$ Alekya Potlapalli ${ }^{1} \quad$ Vidhatri Penmetsa ${ }^{1}$ \\ ${ }^{1}$ Department of Radio-Diagnosis, Basavatarkam Indo-American \\ Cancer Hospital and Research Centre, Hyderabad, Telangana, India \\ Address for correspondence Dr. Rashmi Sudhir, Department of \\ Radio-Diagnosis, Basavatarkam Indo-American Cancer Hospital \\ and Research Centre, Hyderabad 500034, Telangana, India \\ (e-mail: rashmi4210@gmail.com).
}

\begin{abstract}
Keywords

- breast cancer

- biological behavior

- imaging

- pathology

- Indian women

Aims The aim of the study is to evaluate the characteristic imaging features of breast cancer on mammogram, ultrasound, and magnetic resonance imaging (MRI) in women less than 40 years of age and to assess the degree of correlation between clinico-radio-pathological features and biological behavior.

Methods and Materials A prospective observational study on consecutive women under 40 years of age evaluated with ultrasound of breast, digital mammogram, or contrast-enhanced breast MRI, diagnosed with breast cancer on histopathology and molecular analysis done at our center between January and December 2019 were included. Patient demographics, clinical presentation, family history, BRCA mutation status, imaging, pathological findings, and molecular status were determined.

Results Out of 2,470 women diagnosed with breast cancer, 354 (14.3\%) were less than 40 years of age who were included in this study. Mammography showed positive findings in $85 \%$, ultrasonography in $94.3 \%$, and MRI in $96.4 \%$ of women. Majority of the women (69.6\%) presented in the late stage (Stage III and IV) with high-grade carcinoma in $39.5 \%$ and triple-negative breast cancer (TNBC) in $45.7 \%$. Tumors with human epidermal growth factor-2neu expression were associated with the presence of microcalcifications ( $p$-value $=0.006$ ), and TNBC with circumscribed margins or BI-RADS 3/4a category on imaging ( $p$-value $=0.007)$ and high-grade invasive carcinoma compared with others ( $p$-value $<0.0001$ ).

Conclusion The incidence of breast cancer in Indian women less than 40 years of age is relatively high as compared with the West. The detection of breast cancer in young women remains challenging due to dense breast tissue, lower incidence rate, and lack of regular breast screening. While ultrasound is the recommended imaging method for evaluation of breast under the age of 40 years, we found a better characterization of lesions and higher cancer detection rates when they were also evaluated with mammography and MRI.
\end{abstract}

published online July 30,2021
DOI https://doi.org/

$10.1055 / \mathrm{s}-0041-1734342$ ISSN 0971-3026 (c) 2021. Indian Radiological Association.

This is an open access article published by Thieme under the terms of the Creative Commons Attribution-NonDerivative-NonCommercial-License, permitting copying and reproduction so long as the original work is given appropriate credit. Contents may not be used for commercial purposes, or adapted, remixed, transformed or built upon. (https://creativecommons.org/licenses/by-nc-nd/4.0/). Thieme Medical and Scientific Publishers Private Ltd. A-12, Second Floor, Sector -2, NOIDA -201301, India 


\section{Introduction}

Breast cancer is the leading cause of cancer in India and accounts for $27.7 \%$ of all cancers in Indian women. ${ }^{1}$ Breast cancer is usually found in older women. However, there is an apparent higher incidence of breast cancer in younger Indian women compared with the West as nearly $75 \%$ of the Indian population is less than 50 years of age. ${ }^{2}$ The median age of presentation is almost a decade earlier than the western population. It is rare to find breast cancer in women less than 40 years of age in the West and it varies between 2 and 7\% depending on the races and ethnicity. ${ }^{3}$ However, there is a paucity of data available from Indian subcontinent on young breast cancer.

Breast cancer in young women is more likely to be linked with increased familial risk, especially in women carrying BRCA1 or BRCA2 mutation. ${ }^{4}$ Breast cancer diagnosed in younger women tend to present in the late stage with large tumor size, express aggressive behavior, high pathologic grade, rapid proliferation rate, and predominance of triple-negative molecular subtype..$^{5}$ All these features suggest worse prognosis of the disease. Early detection of breast cancer in women less than 40 years of age is more challenging as they are excluded from the standard breast screening program guidelines because of cost-benefit issues and higher mammographic breast density compared with older women.

Our study aimed to evaluate the characteristic imaging features of breast cancer on a digital mammogram (DM), ultrasound (US), and contrast-enhanced breast magnetic resonance imaging (MRI) in women with less than 40 years of age diagnosed with breast cancer and to assess the degree of correlation between clinico-radio-pathological features and biological behavior.

\section{Materials and Methods}

\section{Patients}

A prospective descriptive study was done at a tertiary care cancer hospital in India after approval from the Institutional Scientific Review Board. Consecutive women aged less than 40 years, evaluated with breast US and/or DM or MRI, diagnosed with breast cancer on histopathology and molecular analysis by immunohistochemistry (IHC) examination for estrogen receptor (ER), progesterone receptor (PR), $\mathrm{K}_{\mathrm{i}}-67$ index, and human epidermal growth factor (HER2-neu) expression done at our center between January 2019 and December 2019 were included. Women with a history of prior treatment of breast cancer with surgery, radiotherapy, or chemotherapy were excluded from the study.

On clinical evaluation, data included patient's age, indication for breast imaging, clinical manifestation (palpable mass, pain, nipple discharge, or others), family history (first or second-degree relatives) of breast cancer, personal history (underlying comorbidities, another malignancy or pregnancy) were collected.

\section{Radiological Evaluation}

All the patients were primarily evaluated with an US of breast as mammography is not routinely performed in women less than 40 years of age presenting at our center. In patients with suspicious clinical or US findings or if biopsy yielded malignancy, DM was performed. Breast MRI was done only in small number of cases for high-risk screening, local staging prior to therapeutic planning and as a problem-solving tool when mammography and ultrasonography findings were equivocal. Image interpretation and ultrasonography evaluation were done by two experienced breast radiologists. US examinations were performed on Siemens Acuson S2000 by 12 to $18 \mathrm{MHz}$ high-resolution linear probe, mammography examinations on Senographe Essential DM general electric system and MRI scans were performed on 1.5T (Signa HDx, General Electric Medical system, Milwaukee, Wisconsin, United States) with dedicated breast array coils with patient in prone position. Imaging features were described using 5 th edition ACR BI-RADS (American College of Radiology-Breast Imaging Reporting and Data System). All the BI-RADS 4 and 5 lesions and few of the BI-RADS 3 lesions were biopsied using 14-gauge BARD MAX-CORE disposable biopsy gun under US or mammography (used when only calcifications present, and the lesions were not visible on the US) guidance and samples were sent for histopathology and IHC examinations.

\section{Pathological and Molecular Analysis}

The histopathology slides were examined by experienced breast pathologists and reported according to WHO classification. Tumor grading was done according to the modified Bloom-Richardson grading system. ER, PR, $\mathrm{K}_{\mathrm{i}}-67$, and HER2-neu expressions were determined by IHC examination. In case of equivocal results of HER2-neu on IHC, fluorescence in situ hybridization test was done to confirm.

\section{Statistical Analysis}

Our data were collected on Microsoft Office Excel 2013 and statistical analysis was performed using IBM SPSS version 21(Illinois, Chicago). Student's unpaired $t$-test was used to calculate the difference in continuous variables, and Chi-square test or Fisher's exact test for categorical variables, with $p$-value $<0.05$ was considered significant.

\section{Results}

A total of 2,470 patients including all age groups were diagnosed and treated for breast cancer at our center between January and December 2019 and 354 patients (14.3\%) were less than 40 years of age with a mean age of 34.9 years (range 22-39 years). The patient's clinical and pathological characteristics are summarized in - Table $\mathbf{1}$.

Six patients were pregnant at the time of diagnosis. Thirty-nine patients gave a family history of breast cancer in first- or second-degree relatives. BRCA mutations results were available for five patients and all of them had negative mutations. Of 354 patients, 336 (95\%) were symptomatic 
Table 1 Clinical, pathological, and molecular characteristics of breast cancer in young women

\begin{tabular}{|c|c|}
\hline Number of patients $(n)$ & 354 \\
\hline Age (years) & Mean 34.9 (range 22-39) \\
\hline$<30$ & $33(9.3 \%)$ \\
\hline $30-40$ & $321(90.7 \%)$ \\
\hline \multicolumn{2}{|l|}{ Family history (1st or 2nd degree) } \\
\hline Positive & $39(11 \%)$ \\
\hline Negative & $315(89 \%)$ \\
\hline Symptomatic & $336(95 \%)$ \\
\hline Mass & $280(79 \%)$ \\
\hline Asymptomatic & $18(5 \%)$ \\
\hline \multicolumn{2}{|l|}{ Lymph nodes } \\
\hline Clinical suspicion & $262(74 \%)$ \\
\hline Pathological & $219(62 \%)$ \\
\hline \multicolumn{2}{|l|}{ Clinical stage } \\
\hline 1 & $11(3.1 \%)$ \\
\hline II & $96(27.3 \%)$ \\
\hline III & $163(46.5 \%)$ \\
\hline IV & $81(23.1 \%)$ \\
\hline \multicolumn{2}{|l|}{ Histopathology } \\
\hline IDC \pm DCIS & $328(92.7 \%)$ \\
\hline DCIS & $8(2.3 \%)$ \\
\hline $\begin{array}{l}\text { Others (mucinous, papil- } \\
\text { lary, lobular carcinoma, and } \\
\text { metastasis) }\end{array}$ & $18(6 \%)$ \\
\hline \multicolumn{2}{|l|}{ Histologic grade } \\
\hline $1(\mathrm{G} 1)$ & $8(2.4 \%)$ \\
\hline $2(G 2)$ & $206(58.1 \%)$ \\
\hline $3(G 3)$ & $140(39.5 \%)$ \\
\hline \multicolumn{2}{|l|}{$\begin{array}{l}\text { Biological markers and molecular } \\
\text { subtypes }\end{array}$} \\
\hline ER/PR positive $(\mathrm{LA}+\mathrm{LB})$ & $170(48 \%)$ \\
\hline $\begin{array}{l}\text { (a) ER/PR positive with } \mathrm{K}_{\mathrm{i}}-67< \\
14 \% \text { (LA) }\end{array}$ & $50(14 \%)$ \\
\hline $\begin{array}{l}\text { (b) ER/PR positive with } \mathrm{K}_{\mathrm{i}}-67> \\
\text { 14\% (LB HER-2 negative) }\end{array}$ & $89(25.3 \%)$ \\
\hline HER-2 expression & $53(15 \%)$ \\
\hline $\begin{array}{l}\text { (a) ER/PR and HER-2 positive } \\
\text { (LB HER-2 positive) }\end{array}$ & $31(8.7 \%)$ \\
\hline $\begin{array}{l}\text { (b) ER/PR negative and HER-2 } \\
\text { positive (HER-2 enriched) }\end{array}$ & $22(6.3 \%)$ \\
\hline Triple negative & $162(45.7 \%)$ \\
\hline
\end{tabular}

Abbreviations: DCIS, ductal carcinoma in situ; ER, estrogen receptor; HER2 , human epidermal growth factor receptor; IDC, invasive ductal carcinoma; LA, luminal A molecular subtype; LB, luminal B molecular subtype; $\mathrm{PR}$, progesterone receptor.

and 280 patients (79\%) presented with a palpable lump. In 18 asymptomatic patients (5\%), breast cancers were detected on the mammography or MRI screening done because of positive family history or the patient's request
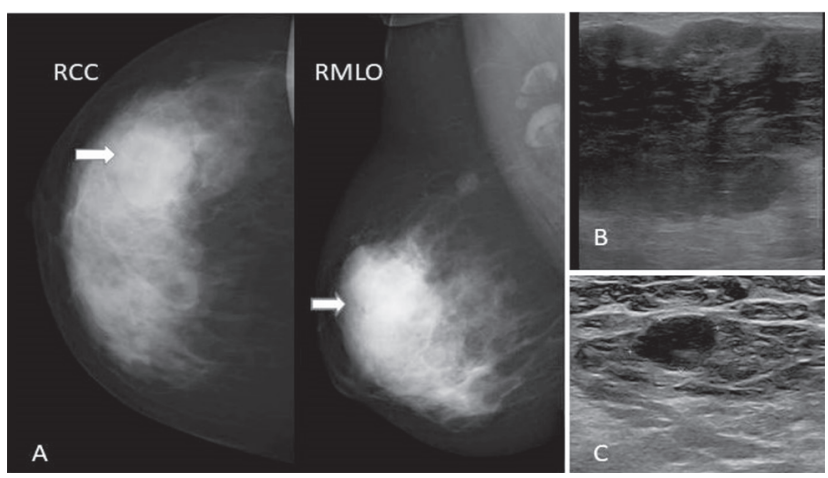

Fig. 1 A 35-year-old woman with stage III (T3N1M0) breast cancer. (A) Digital mammogram, right CC, and MLO view demonstrated extremely dense breast with a rounded hyperdense mass (white arrows) with obscured margins in the upper outer quadrant. (B) US demonstrated a $5.6 \mathrm{~cm}$ large heterogeneous hypoechoic mass at the corresponding location in the right breast. (C) US screening of right axilla revealed a $10-\mathrm{mm}$ rounded lymph node with loss of fatty hilum. The subsequent biopsy yielded triple-negative invasive ductal carcinoma, grade III with lymph node metastasis. CC, craniocaudal; MLO, mediolateral oblique; US, ultrasound.

before marriage or planning a pregnancy. Clinical suspicion of axillary lymphadenopathy was observed in 262 patients (74\%); however, pathological diagnosis of metastatic lymph nodes were seen only in 219 (62\%). Majority of the patients presented in late stage (- Fig. $\mathbf{1}$ ) at the time of diagnosis, stage I in $3.1 \%$, stage II in $27.3 \%$, stage III in $46.5 \%$, and stage IV in $23.1 \%$. Breast conservative surgery was done more frequently (63\%) than the mastectomy (37\%). Infiltrating ductal carcinoma (IDC) with or without ductal carcinoma in situ (DCIS) was the most common histopathological diagnosis of breast cancer (92.7\%), followed by pure DCIS in $2.3 \%$ and others (invasive lobular carcinoma, papillary carcinoma, medullary carcinoma, lymphoma, and adenoid cystic carcinoma) in $6 \%$. Of all the IDCs, $58.1 \%$ were moderate grade (grade II) and $39.5 \%$ were high grade (grade III). On IHC examinations, ER/PR were positive in $48 \%$ of breast tumors with Luminal A (ER/PR positive, $\mathrm{K}_{\mathrm{i}}-67<14 \%$ and HER-2 negative) in $14 \%$ and Luminal B HER-2 negative (ER/PR positive, $\mathrm{K}_{\mathrm{i}}-67>14 \%$ and HER-2 negative) in 25.3\%, Luminal B HER-2 positive (ER/PR and HER-2 positive) in $8.7 \%$, HER-2 enriched (HER-2 positive and ER/PR negative) in $6.3 \%$, and triple-negative breast cancer or TNBC (ER/PR and HER-2 negative) in $45.7 \%$ of patients.

\section{Imaging}

Imaging findings of DM and US are summarized in - Table 2 and MRI in - Table 3.

\section{Digital Mammography}

Digital mammography examinations were performed in 320 of 354 patients with breast cancer as an additional examination to the US if clinical or sonographic features were suspicious of malignancy or after the pathological diagnosis of malignancy. Thirty-four patients could not be evaluated with mammography as the patient underwent breast MRI or excision biopsy or PET/CT following the US examination on breast cancer multidisciplinary team's discretion before 
Table 2 Mammography and ultrasonography features of breast cancer in young women

\begin{tabular}{|l|l|}
\hline Digital mammography $(\boldsymbol{n})$ & 320 \\
\hline Breast density & \\
\hline ACR category A & 0 \\
\hline ACR category B & $21(6.5 \%)$ \\
\hline ACR category C & $180(56.3 \%)$ \\
\hline ACR category D & $119(37.2 \%)$ \\
\hline Positive findings & $272(85 \%)$ \\
\hline No abnormality & $48(15 \%)$ \\
\hline Mass/asymmetry only & $153(47.8 \%)$ \\
\hline Microcalcifications alone & $18(5.6 \%)$ \\
\hline Mass with & $84(26.3 \%)$ \\
\hline microcalcifications & \\
\hline Architectural distortion & $17(5.3 \%)$ \\
\hline Ultrasound (n) & 354 \\
\hline Positive findings & $334(94.3 \%)$ \\
\hline No abnormality & $20(5.7 \%)$ \\
\hline Mass & $325(91.8 \%)$ \\
\hline Circumscribed & $103(31.7 \%)$ \\
\hline Non-circumscribed & $222(68.3 \%)$ \\
\hline Solid (hypo/iso/hyperechoic) & $280(86.2 \%)$ \\
\hline Solid cystic complex & $45(13.8 \%)$ \\
\hline Mean tumor size & $3.4(0.5-9.5) \mathrm{cm}$ \\
\hline Non-mass lesion & $9(2.5 \%)$ \\
\hline BI-RADS category & $250(70.7 \%)$ \\
\hline 1 & $5(1.4 \%)$ \\
\hline 2 & $6(1.7 \%)$ \\
\hline 3 & $9(2.5 \%)$ \\
\hline 4 (4a-35;4b-124; 4c-91) & \\
\hline 5 & \\
\hline
\end{tabular}

Abbreviation: ACR, American College of Radiology; BI-RADS, Breast Imaging Reporting and Data System.

starting the therapy. On DM, breast cancers were suspected in 272 of 320 (85\%) patients.

Majority of the patients (299 out of 320, 93.5\%) had either heterogeneously dense (ACR type C in 56.3\%) or extremely dense (ACR type D in 37.2\%) breast tissue. Mass without microcalcifications was detected in 153 out of 320 patients (47.8\%), mass with microcalcifications in 84 (26.3\%), suspicious microcalcifications alone in 18 patients (5.6\%), and architectural distortion in 17 (5.3\%). False-negative results were observed in 15\% (48 of 320) and all of them had dense breast tissue (ACR type C or type D). All the mammographically occult breast cancers were identified either on US or MRI ( - Fig. 2).

\section{Ultrasound}

Breast US examinations were performed in all 354 patients and breast cancers were suspected in 334 of them (94.3\%) and false negatives in 20 (5.7\%) patients. On US, 325 of 354 patients demonstrated mass lesions (91.8\%), and nine patients (2.5\%) displayed non-mass lesion with or without

Table 3 MRI features of breast cancer in young women

\begin{tabular}{|c|c|}
\hline MRI findings & $n=56(\%)$ \\
\hline \multicolumn{2}{|l|}{ Breast composition } \\
\hline ACR category A or $B$ & $10(17.9)$ \\
\hline ACR category $C$ or $D$ & $46(82.1)$ \\
\hline \multicolumn{2}{|c|}{ Background parenchymal enhancement (BPE) } \\
\hline Minimal & $4(7.1)$ \\
\hline Mild & $26(46.4)$ \\
\hline Moderate & $21(37.5)$ \\
\hline Marked & $5(9)$ \\
\hline Mass & $36(64.3)$ \\
\hline Mass with non-mass enhancement & $10(17.8)$ \\
\hline Non-mass enhancement alone & $8(14.3)$ \\
\hline Negative findings & $2(3.6)$ \\
\hline \multicolumn{2}{|l|}{ Masses $(n=46)$} \\
\hline \multicolumn{2}{|l|}{ Shape } \\
\hline Round & $12(26.1)$ \\
\hline Oval & $8(17.4)$ \\
\hline Irregular & $26(56.5)$ \\
\hline \multicolumn{2}{|l|}{ Margins } \\
\hline Circumscribed & $14(30.4)$ \\
\hline Irregular/spiculated & $32(69.6)$ \\
\hline \multicolumn{2}{|l|}{ Internal enhancement } \\
\hline Homogeneous & $8(17.4)$ \\
\hline Heterogeneous & $29(63)$ \\
\hline $\operatorname{Rim}$ & $9(19.6)$ \\
\hline \multicolumn{2}{|l|}{ Kinetic curve } \\
\hline Type 1 (persistent or progressive) & $5(10.9)$ \\
\hline Type II (plateau) & $22(47.8)$ \\
\hline Type III (wash out) & $19(41.3)$ \\
\hline \multicolumn{2}{|l|}{ Non-mass enhancement $(n=18)$} \\
\hline Focal & $2(11.1)$ \\
\hline Linear & $3(16.7)$ \\
\hline Segmental & $7(38.9)$ \\
\hline Regional & $2(11.1)$ \\
\hline Multiple regions & $4(22.2)$ \\
\hline \multicolumn{2}{|l|}{ Associated features } \\
\hline None & $27(48.2)$ \\
\hline Nipple areolar complex & $5(8.9)$ \\
\hline Skin invasion & $4(7.1)$ \\
\hline Pectoralis invasion & $6(10.7)$ \\
\hline Axillary lymphadenopathy & $24(42.8)$ \\
\hline
\end{tabular}

Abbreviation: ACR, American College of Radiology; MRI, magnetic resonance imaging. 
ductal calcifications. Masses were mostly found to have non-circumscribed (68.3\%) margins and hypoechoic echotexture (86.2\%). Majority of the lesions were assigned either BI-RADS 4 (70.7\%) or BI-RADS 5 (23.7\%) category. The average tumor size on US was $3.4 \mathrm{~cm}$ (range $0.5-9.5 \mathrm{~cm}$ ). Patients with normal US (20 of 354) were detected to have microcalcifications alone in 12 patients ( - Fig. 3 ) and architectural distortion in three patients on mammography ( - Fig. 4 ), and five patients were detected to have suspicious findings on breast MRI. Lesions with positive findings on mammography were diagnosed on stereotactic biopsy. Two of five MRI positive lesions were biopsied after second look US and three patients were biopsied on subsequent follow-up imaging under US or mammography guidance.

In this study cohort, we found 15 patients who were considered to have benign findings (BI-RADS categories 2 or 3 ) on mammography and US both. However, core biopsies were

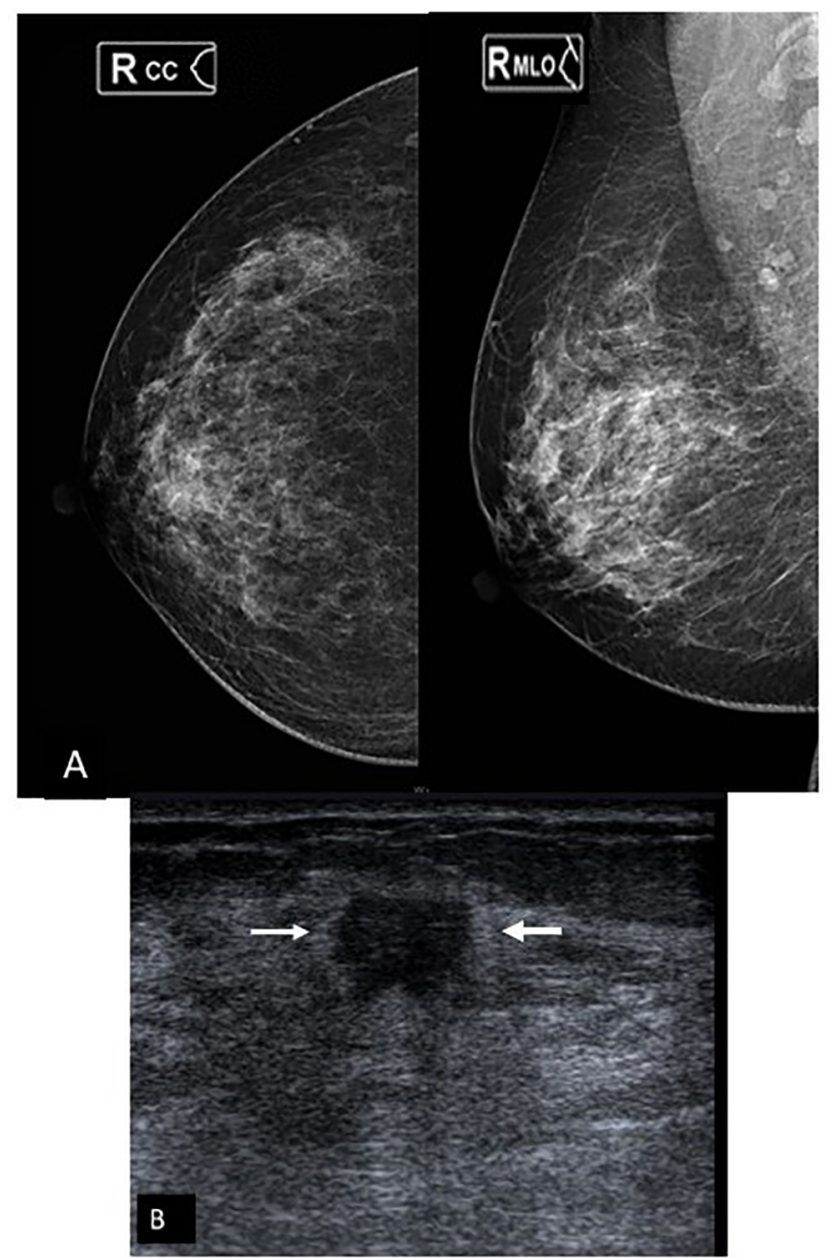

Fig. 2 A 33-year-old-woman with a right breast lump. (A) Digital mammogram, right CC, and MLO views demonstrated heterogeneously dense breast (ACR type $C$ ) with no obvious abnormality. (B) High-resolution ultrasound showed an 11-mm non-circumscribed irregular hypoechoic mass (arrows) in subareolar right breast which subsequently yielded invasive ductal carcinoma on histopathology and ER/PR positive, HER-2neu negative on immunohistochemistry examination. ACR, American College of Radiology; CC, craniocaudal; $E R$, estrogen receptor; $P R$, progesterone receptor; HER, human epidermal growth factor; MLO, mediolateral oblique; $P R$, progesterone receptor. performed on them under US guidance on clinician's request or if the patients were reluctant to come for follow-up.

\section{MRI}

Contrast-enhanced breast MRI was performed in 56 patients with less than 40 years of age during the study period who were diagnosed with breast cancer. MRIs were performed under the indications of high-risk screening

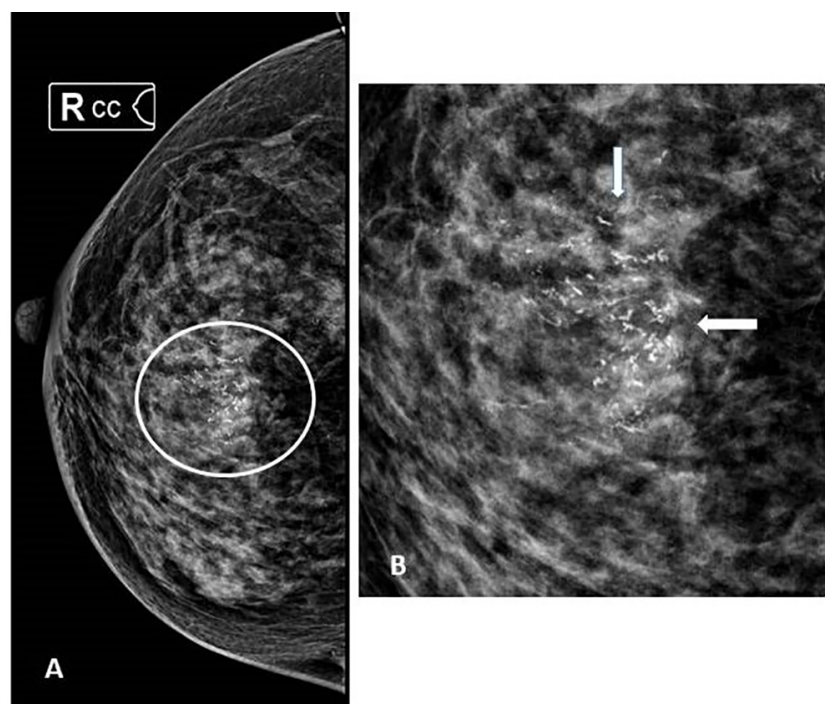

Fig. 3 A 39-year-old woman with a family history of carcinoma breast. High-resolution ultrasound of both the breasts was normal (images not provided here). (A) Digital mammogram, right CC view demonstrated heterogeneously dense breast tissue (ACR category C) with pleomorphic microcalcifications in segmental distribution (encircled), better appreciated in magnified view (B). Subsequent histopathological examination diagnosed high-grade ductal carcinoma in situ (DCIS) without invasion. ACR, American College of Radiology; CC, craniocaudal.

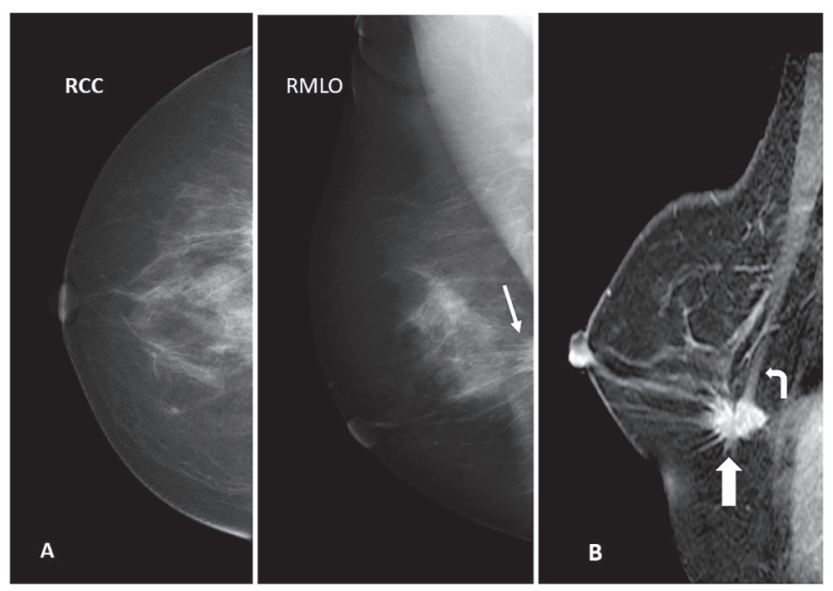

Fig. 4 A 38-year-old woman with right breast pain. Ultrasound was normal (images are nor provided here). (A) Digital mammogram, right CC, and MLO views showed a spiculated lesion with architectural distortion in the posterior third of breast, appreciated only in MLO view (thin white arrow). (B) CE-MRI sagittal section showed homogeneously enhancing spiculated mass (thick white arrow) at the corresponding location with pectoralis muscle (curved arrow) invasion. CC, craniocaudal; CE-MRI, contrast-enhanced magnetic resonance imaging; MLO, mediolateral oblique; 
in 4 , local staging in 36 , and as a problem-solving tool in dense breast in 16 patients. All the MRI features are summarized in - Table 3. Forty-six out of 56 (82.1\%) women showed heterogeneous fibroglandular (ACR category C) or extreme fibroglandular (ACR category D) breast composition on T1W images. Moderate to marked background parenchymal enhancement was seen in 26 (46.5\%) women, which was bilaterally symmetrical. MRI showed positive findings in 54 of 56 patients (96.4\%). The two cases which were negative on MRI showed grouped microcalcifications on mammography and marked background parenchymal enhancement on MRI. Forty-six of 56 women $(82.1 \%)$ were detected to have mass and 10 of 46 masses were associated with non-mass enhancement. The kinetic curve, type I in five (10.9\%), type II in 22 (47.8\%), and type III in $19(41.3 \%)$ of mass lesions. The masses showed circumscribed margins in $30.4 \%$ (14 of 46 ) and irregular or spiculated margins in 69.6\% (32 of 46). Heterogeneous enhancement was observed in 29 (63\%), homogeneous enhancement in eight (17.4\%) and rim enhancement in nine (19.6\%). Eighteen of 56 patients (32.1\%) showed non-mass enhancement; six of 56 patients (10.7\%) showed non-mass enhancement associated with mass lesions and 12 of 56 patients (21.4\%) demonstrated non-mass enhancement alone. The non-mass enhancement was in the segmental distribution in seven of 18 (38.9\%), linear in three (16.7\%), and others (focal, regional, and multiple regions) in eight (44.4\%) patients. Eight out of 56 patients (14.2\%) were detected with additional lesions in the ipsilateral breast and three patients (5.3\%) with contralateral breast lesions which were undetected on pre-MRI DM and ultrasonography.

\section{Clinico-radio-pathological and Molecular Correlation} The correlations between the clinical, radiological, pathological features and molecular subtypes: Luminal A (LA), Luminal B (LB), HER-2 enriched, and TNBC of breast cancers are summarized in - Table $\mathbf{4}$ and - Fig. 5. In this study, there was no significant difference in the mean age of the patients, breast density, and tumor size at the time of diagnosis among different molecular subtypes though the patients with TNBC were found to be relatively younger than the other molecular subtypes ( $p$-value $>0.05$ ). Of the imaging features, microcalcifications detected on mammogram with or without associated mass were significantly correlated with tumors with HER-2 expression $(p=0.006)$ (-Fig. 6), and circumscribed mass or BI-RADS 3 or 4 A mass lesions were associated with TNBC ( $p=0.007)$ as compared with other molecular subtypes which were more frequently found to be non-circumscribed and have irregular margins (-Fig. 7). On MRI, seven out of nine rim enhancing lesions were diagnosed to be triple-negative subtype of breast cancer (-Fig. 8). TNBCs were correlated with a high grade of invasive ductal carcinomas $(p<0.0001)$.

\section{Discussion}

The incidence of breast cancer in women of less than 40 years of age at our center was $14.3 \%$ which is much higher than the incidence rate $(<7 \%)$ reported from the West and falls

Table 4 Correlation between clinical-radio-pathological findings and biological behavior of breast cancer in young women

\begin{tabular}{|c|c|c|c|c|}
\hline $\begin{array}{l}\text { Clinical and imaging features } \\
\text { (MG+US) and pathological } \\
\text { grades }\end{array}$ & $\begin{array}{l}\text { LA \& LB (ER/PR } \\
\text { positive } \pm \text { HER-2 } \\
\text { positive) } n=170\end{array}$ & $\begin{array}{l}\text { HER-2 enriched (HER-2 } \\
\text { positive and ER/PR } \\
\text { negative) } n=22\end{array}$ & $\begin{array}{l}\text { Triple negative (ER/ } \\
\text { PR negative HER-2 } \\
\text { negative) } n=162\end{array}$ & $p$-Value \\
\hline Mean age (years) & $36 \pm 8.5$ & $35.2 \pm 6.4$ & $33 \pm 8.8$ & 0.828 \\
\hline Mean tumor size (cm) & $3.3 \pm 2.7$ & $3.8 \pm 5.1$ & $3.6 \pm 2.3$ & 0.406 \\
\hline \multicolumn{5}{|l|}{ Axillary lymph node metastasis } \\
\hline Negative & $61(35.9 \%)$ & $8(31.8 \%)$ & $66(40.7 \%)$ & \\
\hline Positive & $108(64.1 \%)$ & $15(68.2 \%)$ & $96(59.3 \%)$ & 0.807 \\
\hline \multicolumn{5}{|l|}{ Imaging features } \\
\hline Mass & $65(38.5 \%)$ & $3(13.6 \%)$ & $142(87.6 \%)$ & \\
\hline Mass + calcifications & $60(35.3 \%)$ & $16(63.6 \%)$ & $20(12.4 \%)$ & 0.006 \\
\hline Calcifications only & $14(8.2 \%)$ & $5(22.7 \%)$ & 0 & \\
\hline Circumscribed margins & $13(7.5 \%)$ & $8(21 \%)$ & $86(58.4 \%)$ & 0.004 \\
\hline Non-circumscribed margins & 157 (92.5\%) & $14(79 \%)$ & $64(41.6 \%)$ & \\
\hline \multicolumn{5}{|l|}{ BI-RADS } \\
\hline $3,4 a$ & $12(7 \%)$ & $6(27.3 \%)$ & $26(16 \%)$ & 0.007 \\
\hline $4 b, 4 c, 5$ & $158(93 \%)$ & $16(72.7 \%)$ & $136(84 \%)$ & \\
\hline \multicolumn{5}{|l|}{ Histologic grade } \\
\hline Grade 1 and 2 & 147 (86.5\%) & $12(54.5 \%)$ & $55(34 \%)$ & 0.569 \\
\hline Grade 3 & $23(13.5 \%)$ & $10(45.5 \%)$ & $107(66 \%)$ & 0.0001 \\
\hline
\end{tabular}

Abbreviations: ER, estrogen receptor; HER, human epidermal growth; LA, luminal A molecular subtype; LB, luminal B molecular subtype; PR, progesterone receptor; US, ultrasound. 
Age distribution (Mean age 34.9 years; Range 22-39years)

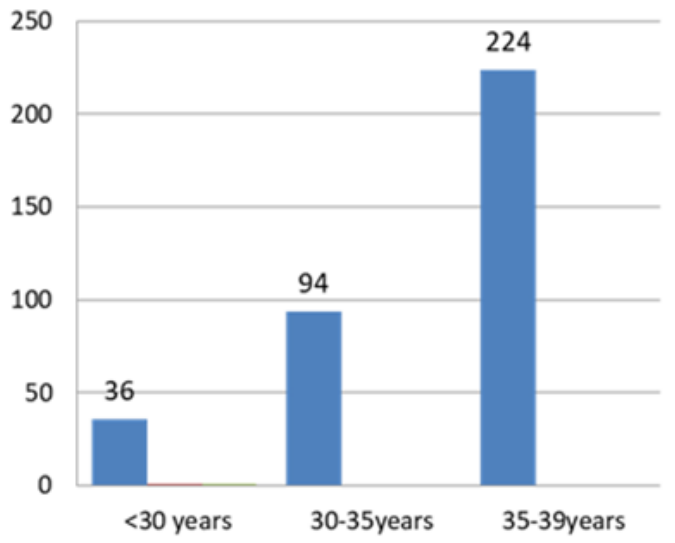

Correlation between the mean age and molecular subtypes of breast cancer in young women ( $<40$ years)

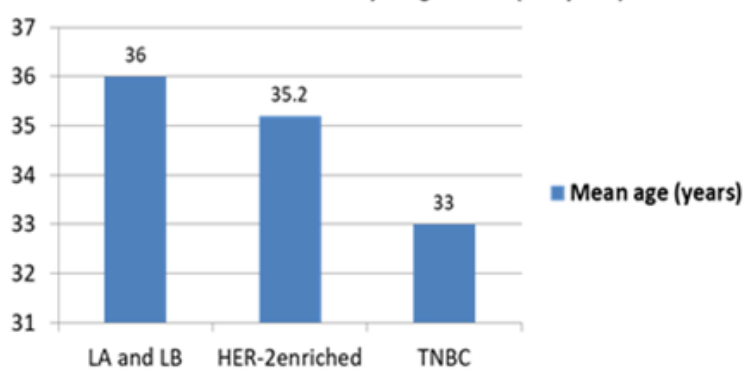

ACR breast density distribution in young women ( $<40$ years)

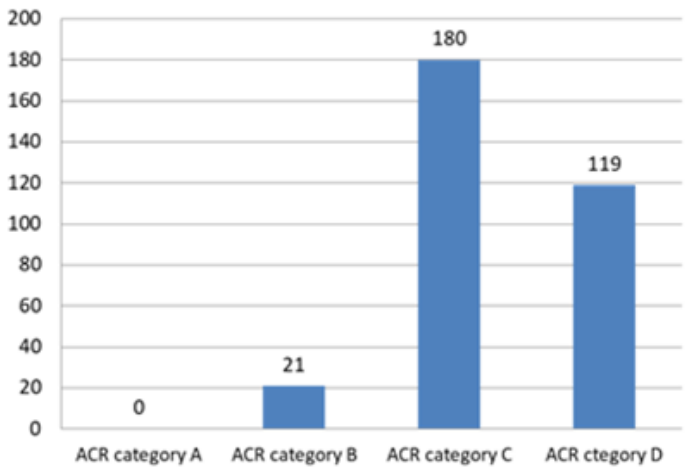

Radio-pathological and biological behaviour correlation

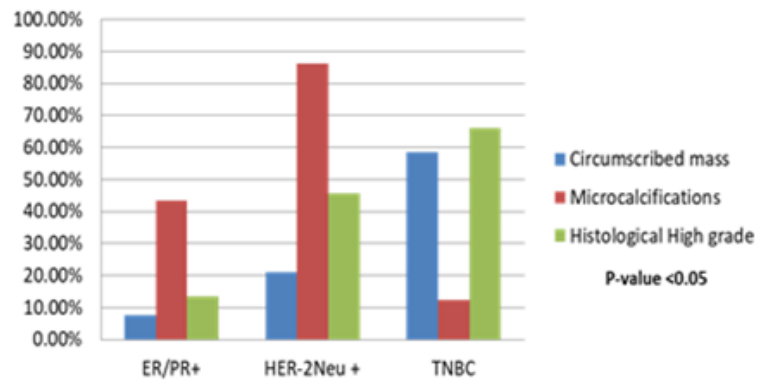

Fig. 5 The graphical representation of age distribution, breast density, and correlation between the clinical, pathological, and biological behavior of breast cancer in women $<40$ years of age.

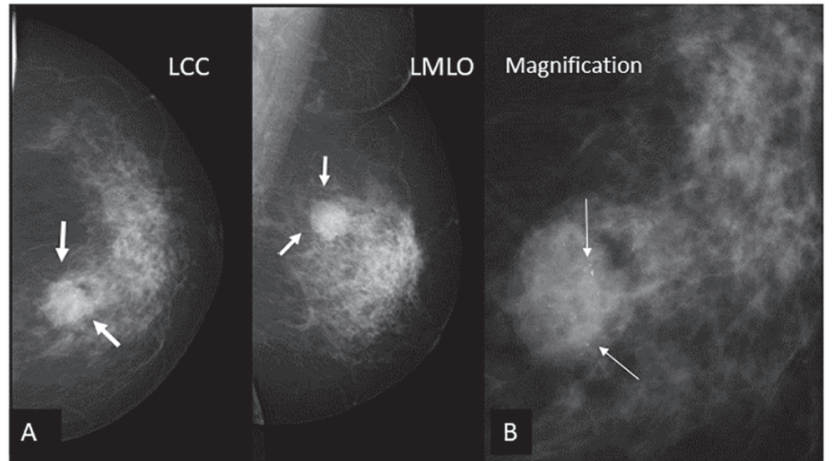

Fig. 6 A 34-year-old woman with HER-2 enriched subtype of invasive ductal carcinoma, grade II in the right breast. (A) A rounded hyperdense mass with irregular margins in upper inner quadrant (thick white arrows). (B) Magnification view of the mass showed pleomorphic microcalcifications (thin white arrows) within the mass. HER, human epidermal growth factor.

within the range (12-18\%) reported among Asian women. ${ }^{3,6,7}$ The higher incidence rate could be attributed to the fact that more than three-fourths of the Indian population is under 50 years of age and a possible selection bias, as this study was done at a tertiary care referral cancer hospital. Majority of the patients in this study were symptomatic with $79 \%$ of them presenting with a palpable lump with mean tumor size of $3.4 \mathrm{~cm}$. They were more frequently found to have moderate $(58.1 \%)$ or high-grade $(39.5 \%)$ invasive ducal carcinoma (92.7\%) and in advanced stage (69.6\% in stage III and IV), which were concordant with previously reported studies. ${ }^{8,9}$ Invasive lobular, papillary, and mucinous carcinoma are significantly less common in younger women compared with older women. ${ }^{10}$ Diagnosis of breast cancer in young women may be difficult and diagnosed late as breast lumps are more likely to be interpreted as benign lesions because of lower incidence rate of malignancy compared with older women. In this study cohort, family history of breast or ovarian cancer in first- or second-degree relative was present only in $9 \%$ of patients which was significantly less than the association of $34 \%$ reported in young British women. ${ }^{11}$

In young women, mammography examinations are considered less sensitive for breast cancer diagnosis because of the dense breast which has been hypothesized to be the cause for rapidly growing and aggressive tumors in this age group. In the current study, DM was performed to examine the breasts and the sensitivity rate was $85 \%$, which is relatively higher for the mammography examinations, but still falls within the previously reported range of 40 to $92 \% .8,9,12,13$ The higher sensitivity could be explained by the recent technological advancements in the form of DM, which aided significant improvement in the evaluation of dense breasts 


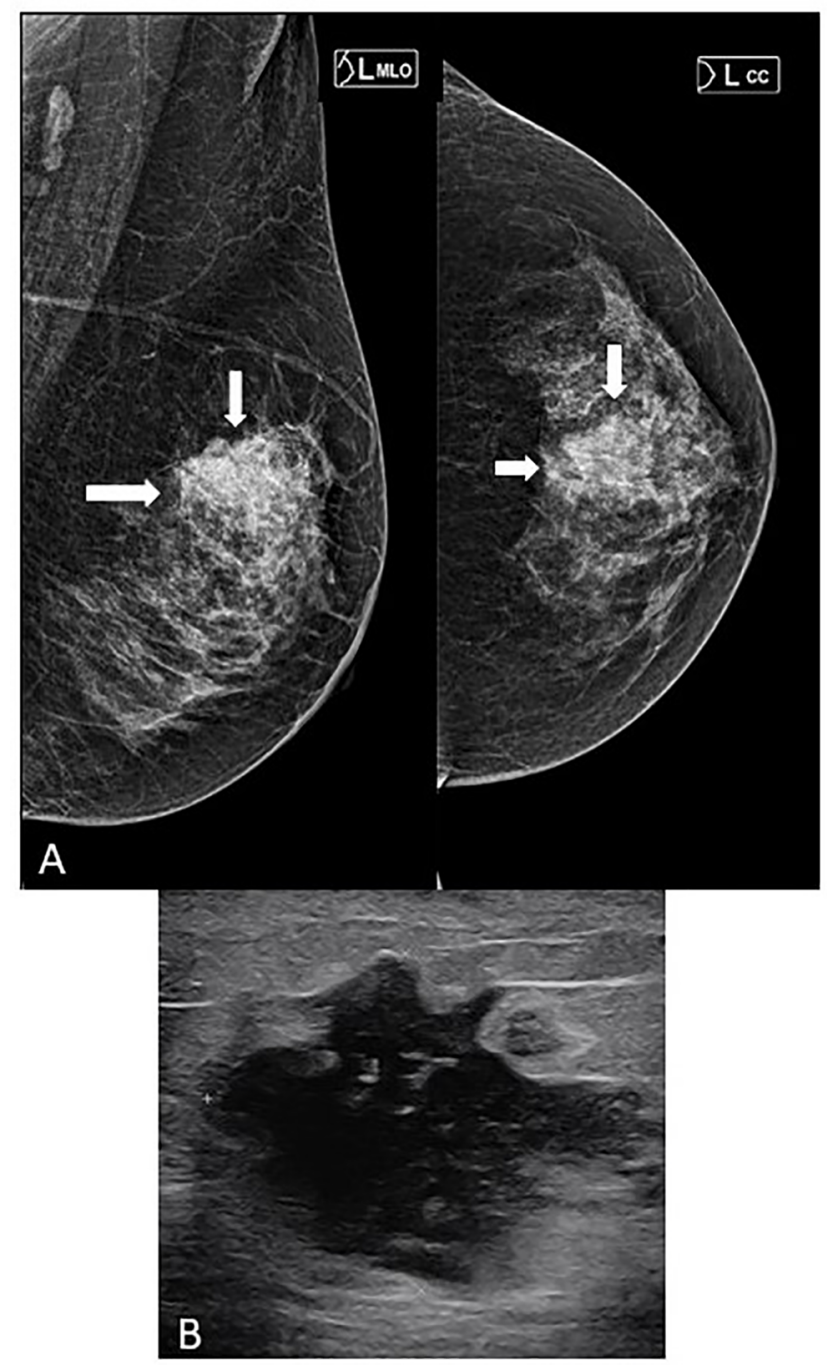

Fig. 7 A 34-year-old woman with luminal type (ER/PR+ and HER-2neu negative) of invasive breast cancer, grade II. (A) Digital mammography, left CC, and MLO views revealed heterogeneously dense breast (ACR type C) with a noncircumscribed irregular hyperdense mass (arrows) corresponding to $32 \mathrm{~mm}$, hypoechoic mass with angular margins on ultrasound (B). ACR, American College of Radiology; CC, craniocaudal; ER, estrogen receptor; PR, progesterone receptor; HER, human epidermal growth factor; MLO, mediolateral oblique; PR, progesterone receptor.

and patient selection bias as the DM examinations were performed in patients with suspicious clinical or US findings or if biopsy yielded malignancy prior to starting the therapy. In this study, DM could detect microcalcifications either alone or with associated mass in $31.9 \%$ and architectural distortion in $5.3 \%$ of cases. The false-negative cases on mammogram were mainly due to obscuration of lesions by the superimposition of breast tissue in the heterogeneously dense or extremely dense breast, which was seen in $93.5 \%$ of our patients. It is a known fact that dense breast and young age are the strong factors associated with reduced sensitivity of mammogram in diagnosing breast cancer. ${ }^{14,15}$ Microcalcifications alone were detected in $5.6 \%$ of patients who were either diagnosed as DCIS alone or with a small invasive component. All the cases with pure microcalcifications were either missed on US

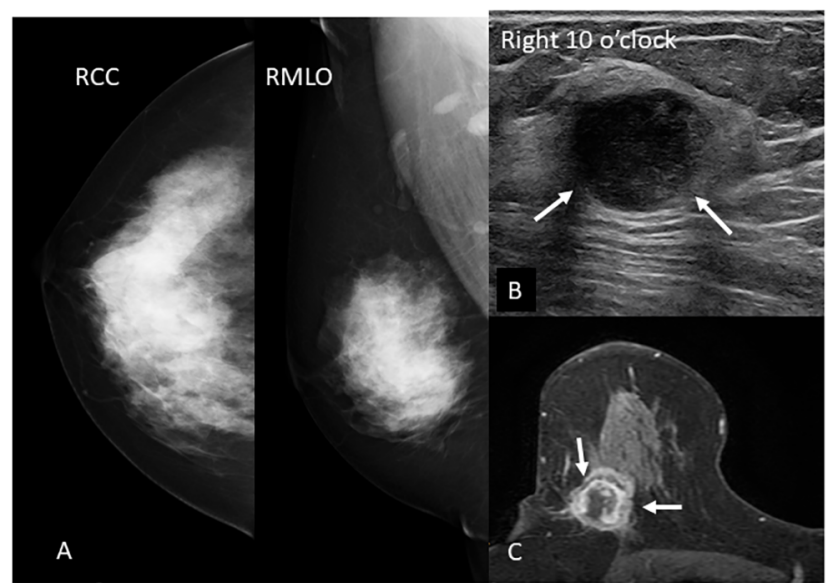

Fig. 8 A 37-year-old woman with a triple-negative (ER/PR and HER-2 negative) invasive ductal carcinoma, grade III in the right breast. (A) Digital mammography right CC and MLO views showed extremely dense breast with no obvious abnormality. (B) Ultrasound demonstrated a well-circumscribed rounded hypoechoic mass with posterior acoustic enhancement at 10 o'clock right breast. (C) On contrast-enhanced breast MRI, the corresponding mass showed rim enhancement with central necrosis. CC, craniocaudal; ER, estrogen receptor; PR, progesterone receptor; HER, human epidermal growth factor; MLO, mediolateral oblique; MRI, magnetic resonance imaging; PR, progesterone receptor.

examinations or showed some equivocal features. Thus, DM may play an important role in symptomatic young patients or with suspicious or equivocal US findings.

Ultrasound is the primary imaging modality for the initial evaluation of breast lesions in young women. In this study, the US showed positive findings in $94.3 \%$ of the patients. Similar to previously published studies, breast lesions on the US were more frequently non-circumscribed (68.3\%) and hypoechoic solid (86.2\%) masses. ${ }^{8,9}$ On combining mammography and US findings, majority of the women with breast cancer in this study were assigned either BI-RADS 4 (70.7\%) and $5(23.7 \%)$ category, which suggested a very strong correlation with histological diagnosis ( $p$-value 0.0001 ).

Contrast-enhanced breast MRIs were performed for local staging of breast cancer prior to therapeutic planning, screening of high-risk women, and as a problem-solving tool when findings were inconclusive on conventional imaging. On MRI, the breast cancers showed enhancing masses in $82.1 \%$ of patients with or without associated non-mass enhancement. Masses were mostly irregular in shape (56.5\%) and showed heterogeneous (63\%) or rim enhancement (19.6\%). Non-mass enhancements alone were detected in $14.3 \%$ of patients and more frequently in segmental distribution. Fifteen of 18 non-mass enhancing lesions (83.3\%) were diagnosed to have DCIS component on final histopathology. Reports have shown that MRI has the highest sensitivity to detect breast cancer, additional lesions in the same or contralateral breast and to assess the local extent of the disease. ${ }^{16,17}$ Study reported by Kuhl et al showed that sensitivity of mammography, ultrasonography, and MRI in asymptomatic women with high risk for breast cancer was 33\%, 40\%, and $91 \%$, respectively. ${ }^{16}$ Concordant to the study published by previous 
investigators, our study also showed very high performance rate of MRI in detecting histologically established solitary, multifocal, multicentric, or bilateral breast cancers.

Breast cancers in young women are known to demonstrate aggressive behavior and worse prognosis as compared with older women. ${ }^{18,19}$ These women often present in higher stage with high-grade tumor and high proliferation index. ${ }^{18-22}$ In our study, $69.6 \%$ of patients were diagnosed in advanced stage (stage III and IV), 39.5\% with high grade (grade III), 34\% with high proliferation index, and $46 \%$ demonstrated triple-negative molecular subtype (ER/PR negative and HER-2 negative) of breast cancer, which was concordant with the studies reported in the literature. $8,9,23-26$ TNBC was frequently associated with high-grade tumor $(66 \%, p$-value $<0.0001)$, circumscribed mass $(58.4 \%$, $p$-value $<0.004$ ) and assigned as BI-RADS 3/4a category lesions $(p<0.007){ }^{24,26,27}$ Luminal B was the most prevalent molecular phenotype (ER/PR positive with KI-67 $>14 \%$ or ER/PER positive and HER-2 positive) in our study (42.7\%) concordant with the study published by Bacchi et al. ${ }^{28}$ HER-2 enriched (ER/PR negative and HER-2 positive) molecular type was present only in 22 patients (6.2\%). Similar to postmenopausal women, HER-2 enriched tumors were associated with the presence of mammographically detected microcalcifications (63.6\%) with $p$-value $<0.006{ }^{24,28-30}$ Unlike older women, Luminal A molecular type of breast cancers were less prevalent (14\%) in our study cohort. Similar to previously reported studies, ER/PR positive tumors (Luminal A and B molecular subtypes) were more frequently found to be non-circumscribed mass with irregular or spiculated margins due to stromal reaction and assigned with ACR BI-RADS $4 \mathrm{~b}$ or above category. ${ }^{31}$

There were a few limitations in this study. First, this study was conducted at a single center, which could have led to selection bias. Second, the number of patients evaluated with breast MRI was very small, hence a definitive conclusion needs larger studies. Third, BRCA analyses were done only for five patients.

\section{Conclusion}

The incidence of breast cancer in Indian women less than 40 years of age is relatively higher as compared with the West. Since there is lower prevalence of breast cancer and no recommendation of regular breast screening in this age group, most of them present with palpable breast lump in advanced stage. Absence of positive family history should not reduce the degree of suspicion as majority of these patients do not have a positive family history of breast cancer or ovarian cancer. Though US is the recommended imaging method for evaluation of breast in women under the age of 40 years, we found a better characterization of lesions and higher cancer detection rates when they were also evaluated with mammography and MRI. The histological and biological behavior of the breast cancers was well correlated with the imaging features.

\section{Financial Support and Sponsorship}

None.

\section{Conflict of Interest}

None declared.

\section{References}

1 Bray F, Ferlay J, Soerjomataram I, Siegel RL, Torre LA, Jemal A. Global cancer statistics 2018: GLOBOCAN estimates of incidence and mortality worldwide for 36 cancers in 185 countries. CA Cancer J Clin 2018;68(6):394-424

2 Parmar V. Rising incidence of breast cancer in the young fertile Indian population-a reality check. Indian J Surg Oncol 2018;9(3):296-299

3 Jemal A, Siegel R, Ward E, et al. Cancer statistics, 2008. CA Cancer J Clin 2008;58(2):71-96

4 Antoniou A, Pharoah PD, Narod S, et al. Average risks of breast and ovarian cancer associated with BRCA1 or BRCA2 mutations detected in case series unselected for family history: a combined analysis of 22 studies. Am J Hum Genet 2003;72(5):1117-1130

5 Lee H-B, Han W. Unique features of young age breast cancer and its management. J Breast Cancer 2014;17(4):301-307

6 Anders CK, Johnson R, Litton J, Phillips M, Bleyer A. Breast cancer before age 40 years. Semin Oncol 2009;36(3):237-249

7 Teo SY, Chuwa E, Latha S, Lew YL, Tan YY. Young breast cancer in a specialised breast unit in Singapore: clinical, radiological and pathological factors. Ann Acad Med Singap 2014;43(2):79-85

8 Bullier B, MacGrogan G, Bonnefoi $\mathrm{H}$, et al. Imaging features of sporadic breast cancer in women under 40 years old: 97 cases. Eur Radiol 2013;23(12):3237-3245

9 An YY, Kim SH, Kang BJ. Characteristic features and usefulness of MRI in breast cancer in patients under 40 years old: correlations with conventional imaging and prognostic factors. Breast Cancer 2014;21(3):302-315

10 Kwong A, Cheung P, Chan S, Lau S. Breast cancer in Chinese women younger than age 40: are they different from their older counterparts? World J Surg 2008;32(12):2554-2561

11 Eccles BK, Copson ER, Cutress RI, et al; POSH Study Steering Group. Family history and outcome of young patients with breast cancer in the UK (POSH study. $\mathrm{Br} \mathrm{J}$ Surg 2015;102(8):924-935

12 Foxcroft LM, Evans EB, Porter AJ. The diagnosis of breast cancer in women younger than 40. Breast 2004;13(4):297-306

13 Eugenio DSG, Souza JA, Chojniak R, Bitencourt A, Graziano L, Marques EF. Breast cancer diagnosed before the 40 years: imaging findings and correlation with histology and molecular subtype. Appl Cancer Res 2017;37:16-29

14 Kerlikowske K, Grady D, Barclay J, Sickles EA, Ernster V. Effect of age, breast density, and family history on the sensitivity of first screening mammography. JAMA 1996;276(1):33-38

15 Carney PA, Miglioretti DL, Yankaskas BC, et al. Individual and combined effects of age, breast density, and hormone replacement therapy use on the accuracy of screening mammography. Ann Intern Med 2003;138(3):168-175

16 Kuhl CK, Schrading S, Leutner CC, et al. Mammography, breast ultrasound, and magnetic resonance imaging for surveillance of women at high familial risk for breast cancer. J Clin Oncol 2005;23(33):8469-8476

17 Sardanelli F, Giuseppetti GM, Panizza P, et al. Italian Trial for Breast MR in Multifocal/Multicentric Cancer. Sensitivity of MRI versus mammography for detecting foci of multifocal, multicentric breast cancer in fatty and dense breasts using the whole-breast pathologic examination as a gold standard. AJR Am J Roentgenol 2004;183(4):1149-1157

18 Han W, Kim SW, Park IA, et al. Young age: an independent risk factor for disease-free survival in women with operable breast cancer. BMC Cancer 2004;4:82 
19 Maggard MA, O'Connell JB, Lane KE, Liu JH, Etzioni DA, Ko CY. Do young breast cancer patients have worse outcomes? J Surg Res 2003;113(1):109-113

20 Anders CK, Hsu DS, Broadwater G, et al. Young age at diagnosis correlates with worse prognosis and defines a subset of breast cancers with shared patterns of gene expression. J Clin Oncol 2008;26(20):3324-3330

21 Kroman N, Jensen MB, Wohlfahrt J, Mouridsen HT, Andersen PK, Melbye M. Factors influencing the effect of age on prognosis in breast cancer: population-based study. BMJ 2000;320(7233):474-478

22 Rapiti E, Fioretta G, Verkooijen HM, et al. Survival of young and older breast cancer patients in Geneva from 1990 to 2001. Eur J Cancer 2005;41(10):1446-1452

23 Collins LC, Marotti JD, Gelber S, et al. Pathologic features and molecular phenotype by patient age in a large cohort of young women with breast cancer. Breast Cancer Res Treat 2012;131(3):1061-1066

24 Rashmi S, Kamala S, Murthy SS, Kotha S, Rao YS, Chaudhary KV. Predicting the molecular subtype of breast cancer based on mammography and ultrasound findings. Indian J Radiol Imaging 2018;28(3):354-361

25 O'Brien KM, Cole SR, Tse CK, et al. Intrinsic breast tumor subtypes, race, and long-term survival in the Carolina Breast Cancer Study. Clin Cancer Res 2010;16(24):6100-6110
26 Caldarella A, Crocetti E, Bianchi S, et al. Female breast cancer status according to ER, PR and HER2 expression: a population-based analysis. Pathol Oncol Res 2011;17(3):753-758

27 Carvalho FM, Bacchi LM, Santos PP, Bacchi CE. Triplenegative breast carcinomas are a heterogeneous entity that differs between young and old patients. Clinics (São Paulo 2010;65(10):1033-1036

28 Bacchi LM, Corpa M, Santos PP, Bacchi CE, Carvalho FM. Estrogen receptor-positive breast carcinomas in younger women are different from those of older women: a pathological and immunohistochemical study. Breast 2010;19(2):137-141

29 Di Nubila B, Cassano E, Urban LA, et al. Radiological features and pathological-biological correlations in 348 women with breast cancer under 35 years old. Breast 2006;15(6):744-753

30 Elias SG, Adams A, Wisner DJ, et al. Imaging features of HER2 overexpression in breast cancer: a systematic review and meta-analysis. Cancer Epidemiol Biomarkers Prev 2014;23(8):1464-1483

31 Patel TA, Puppala M, Ogunti RO, et al. Correlating mammographic and pathologic findings in clinical decision support using natural language processing and data mining methods. Cancer 2017;123(1):114-121 